\title{
UNIQUE SOLVABILITY OF AN EXTENDED STIELTJES MOMENT PROBLEM
}

\author{
OLAV NJÅSTAD
}

(Communicated by Paul S. Muhly)

\begin{abstract}
Let $a_{1}, \ldots, a_{p}$ be given real numbers ordered by size, and let $\{\alpha, \beta]$ be a real interval disjoint from the set $\left\{a_{1}, \ldots, a_{p}\right\}$. Let $\left\{c_{j}^{(i)}: j=\right.$ $1,2, \ldots\}, i=1, \ldots, p$, be sequences of real numbers and $c_{0}$ be a real number. The extended Stieltjes moment problem is to find a distribution function $\psi$ with all its points of increase in $[\alpha, \beta]$ such that

$$
\int_{\alpha}^{\beta} d \psi(t)=c_{0}, \quad \int_{\alpha}^{\beta} \frac{d \psi(t)}{\left(t-a_{i}\right)^{j}}=c_{j}^{(i)}, \quad i=1, \ldots, p, j=1,2, \ldots
$$

Necessary and sufficient conditions for the existence of a unique solution of the problem are given. Orthogonal $R$-functions and Gaussian quadrature formulas play important roles in the proof.
\end{abstract}

1. Introduction. By a distribution function we shall mean a real-valued, bounded, nondecreasing function $\psi(t)$ with an infinite number of points of increase. By its Stieltjes transform we mean the function $\hat{\psi}(z)=\int_{-\infty}^{\infty} d \psi(t) /(t-z)$. If all the points of increase of $\psi$ lie in an interval $I$, then $\hat{\psi}(z)$ is an analytic function in $C-\bar{I}$.

Let $a_{1}, a_{2}, \ldots, a_{p}$ be distinct real numbers, ordered by size. We shall call the real interval $[\alpha, \beta]$ a Stieltjes interval for the point set $\left\{a_{1}, \ldots, a_{p}\right\}$ if $(\alpha, \beta) \cap$ $\left\{a_{1}, \ldots, a_{p}\right\}=\varnothing$. (Intervals of the form $(-\infty, \beta]$ and $[\alpha, \infty)$ are allowed.)

Let $\left\{c_{j}^{(i)}: j=1,2, \ldots\right\}, i=1, \ldots, p$, be given sequences of real numbers and $c_{0}$ be a real number. The extended Stieltjes moment problem (ESMP) is defined as follows: Find a distribution function $\psi(t)$ with all its points of increase in a given Stieltjes interval $[\alpha, \beta]$ such that

$$
\int_{\alpha}^{\beta} d \psi(t)=c_{0}, \quad \int_{\alpha}^{\beta} \frac{d \psi(t)}{\left(t-a_{i}\right)^{j}}=c_{j}^{(i)}, \quad j=1,2, \ldots, i=1, \ldots, p .
$$

In $[3,4]$ we studied the extended Hamburger moment problem (EHMP), which consists of finding a distribution function with the same properties as above, except the requirements that the points of increase lie in a given interval. Conditions for existence of a solution of the ESMP were briefly discussed in [5]. In this paper we give necessary and sufficient conditions for the existence of a unique solution of the problem. Since the tools used in proving the existence of solutions also are involved in the discussion of the uniqueness question, we give a very brief sketch of the existence proof here.

Received by the editors September 8, 1986.

1980 Mathematics Subject Classification (1985 Revision). Primary 30E05; Secondary 42 C05. $K e y$ words and phrases. Moment problems, orthogonal $R$-functions. 
The treatment of the uniqueness problem is related to the treatment of the uniqueness problem for the strong Stieltjes moment problem, given in terms of continued fractions in $[\mathbf{1}, \mathbf{2}]$. For the classical Stieltjes moment problem, see e.g. $[6,7]$.

2. Orthogonal $R$-functions. Let $R$ denote the linear space consisting of all functions of the form

$$
R(z)=\alpha_{0}+\sum_{i=1}^{p} \sum_{j=1}^{N_{i}} \frac{\alpha_{i j}}{\left(z-a_{i}\right)^{j}}, \quad \alpha_{0}, \alpha_{i j} \in \mathbf{C} .
$$

Elements of $R$ are called $R$-functions. We denote by $R_{\mathbf{R}}$ the real space of all $R$ functions with real coefficients. A function $R$ belongs to $R$ iff it can be written in the form $R(z)=P(z) / Q(z)$, where $Q$ is a polynomial with all its zeros among the points $a_{1}, \ldots, a_{p}$, and where $P$ is a polynomial with $\operatorname{deg} P \leq \operatorname{deg} Q$. The spaces $R$ and $R_{\mathbf{R}}$ are closed under multiplication.

Every natural number $n$ has a unique decomposition $n=q_{n} p+r_{n}, 1 \leq r_{n} \leq p$. We write $q=q_{n}, r=r_{n}$. We denote by $R_{n}$ the space of $R$-functions of the form

$$
R(z)=\alpha_{0}+\sum_{i=1}^{r} \sum_{j=1}^{q+1} \frac{\alpha_{i j}}{\left(z-a_{i}\right)^{j}}+\sum_{i=r+1}^{p} \sum_{j=1}^{q} \frac{\alpha_{i j}}{\left(z-a_{i}\right)^{j}} .
$$

Let $\Phi$ denote the linear functional defined on $R$ by

$$
\Phi\left(\alpha_{0}+\sum_{i=1}^{p} \sum_{j=1}^{N_{i}} \frac{\alpha_{i j}}{\left(z-a_{i}\right)^{j}}\right)=\alpha_{0} c_{0}+\sum_{i=1}^{p} \sum_{j=1}^{N_{i}} \alpha_{i j} c_{j}^{(i)} .
$$

We shall say that $\Phi$ is positive on an interval $[a, b]$ if $\Phi(R)>0$ for every $R \in R$ such that $R(t) \geq 0$ and $R(t) \not \equiv 0$ for $t \in(a, b)$. It follows immediately that a necessary condition for the ESMP for a given Stieltjes interval $[\alpha, \beta]$ to have a solution is that $\Phi$ is positive on $[\alpha, \beta]$. We shall in the following assume that this condition is satisfied.

If the functional $\Phi$ is positive on some interval $[\alpha, \beta]$, it is positive on $(-\infty, \infty)$, and therefore gives rise to an inner product $\langle$,$\rangle defined by \langle R, S\rangle=\Phi(R \cdot S)$. By applying the Gram-Schmidt procedure to the sequence

$$
\left\{1, \frac{1}{\left(z-a_{1}\right)}, \ldots, \frac{1}{\left(z-a_{p}\right)}, \frac{1}{\left(z-a_{1}\right)^{2}}, \ldots, \frac{1}{\left(z-a_{p}\right)^{2}}, \frac{1}{\left(z-a_{1}\right)^{3}}, \ldots\right\}
$$

we obtain an orthonormal sequence $\left\{Q_{n}\right\}$ of $R$-functions. We note that $\left\langle Q_{n}, R\right\rangle=0$ for every $R \in R_{n-1}$. Furthermore $Q_{n}$ may be written as $Q_{n}(z)=V_{n}(z) / N_{n}(z)$, where

$$
N_{n}(z)=\left(z-a_{1}\right)^{q+1} \cdots\left(z-a_{r}\right)^{q+1}\left(z-a_{r+1}\right)^{q} \cdots\left(z-a_{p}\right)^{q},
$$

and $V_{n}(z)$ is a polynomial of degree at most $n$.

3. Existence of solutions. When $\Phi$ is positive on a Stieltjes interval $[\alpha, \beta]$, the polynomial $V_{n}(z)$ is of degree $n$, and has $n$ real simple zeros in $(\alpha, \beta)$ (cf. [5], see also [3]). This can be seen as follows: Let $t_{1}, \ldots, t_{\lambda}$ be all the zeros of odd order of $V_{n}(z)$ in $(\alpha, \beta)$. If $\lambda<n$, then the function

$$
T_{n}(z)=\frac{\left(z-t_{1}\right) \cdots\left(z-t_{\lambda}\right)\left(z-a_{r}\right)}{N_{n}(z)}
$$


belongs to $R_{n-1}$, hence $\Phi\left(Q_{n} T_{n}\right)=0$. On the other hand the function

$$
T_{n}(z) Q_{n}(z)=\frac{\left(z-t_{1}\right) \cdots\left(z-t_{\lambda}\right) V_{n}(z)\left(z-a_{r}\right)}{N_{n}(z)^{2}}
$$

has a fixed sign in $(\alpha, \beta)$, since $\left(z-a_{r}\right)$ does not change sign in $(\alpha, \beta)$. Thus $\Phi\left(Q_{n} T_{n}\right) \neq 0$, which is a contradiction. Consequently $\lambda=n$, and the result follows.

With the zeros $t_{1}^{(n)}, \ldots, t_{n}^{(n)}$ of $V_{n}(z)$ (or equivalently of $\left.Q_{n}(z)\right)$ there is associated a Gaussian quadrature formula. There exist positive weights $\lambda_{n, 1}, \ldots, \lambda_{n, n}$ such that

$$
\Phi(R)=\sum_{k=1}^{n} \lambda_{n, k} R\left(t_{k}^{(n)}\right)
$$

for every $R$ of the form (2.1), where $N_{i} \leq 2 q+2$ for $i<r, N_{i} \leq 2 q$ for $i>r$, and $N_{r} \leq 2 q+1$ (see [3, Theorem 4.2, Proposition 4.4; 4, Theorems 2.6, 2.7]). For given $i, j$ we may then write

$$
c_{j}^{(i)}=\Phi\left(\frac{1}{\left(z-a_{i}\right)^{j}}\right)=\sum_{k=1}^{n} \lambda_{n, k} \frac{1}{\left(t_{k}^{(n)}-a_{i}\right)^{j}}
$$

for sufficiently large $n$. Thus we may also write $c_{j}^{(i)}=\int_{\alpha}^{\beta} d \phi_{n}(t) /\left(t-a_{i}\right)^{j}$, where the nondecreasing, uniformly bounded functions $\phi_{n}(t)$ are defined by $\phi_{n}(t)=$ $\sum\left\{\lambda_{n, k}: t_{k}^{(n)} \leq t\right\}$. Application of Helly's selection and convergence theorems show that the sequence $\left\{\phi_{n}(t)\right\}$ contains at least one subsequence converging to a distribution function $\phi$, and the limit function $\phi$ of every such convergent subsequence has all its points of increase in $(\alpha, \beta)$ and satisfies

$$
\int_{\alpha}^{\beta} d \phi(t)=c_{0}, \quad \int_{\alpha}^{\beta} \frac{d \phi(t)}{\left(t-a_{i}\right)^{j}}=c_{j}^{(i)}, \quad j=1,2, \ldots, i=1, \ldots, p .
$$

(For more details, see [3].) Thus we have seen

THEOREM 1. A necessary and sufficient condition for the ESMP on the given Stieltjes interval $[\alpha, \beta]$ to have a solution is that $\Phi$ is positive on $[\alpha, \beta]$.

4. Uniqueness of solution. We define the $R$-function $P_{n}$ associated with $Q_{n}$ by

$$
P_{n}(z)=\Phi_{t}\left(\frac{Q_{n}(t)-Q_{n}(z)}{t-z}\right) .
$$

(The subscript $t$ indicates that the functional operates on its argument as a function of $t$.) The function $P_{n}(z)$ can be written in the form $P_{n}(z)=U_{n}(z) / N_{n}(z)$ where $U_{n}(z)$ is a polynomial of degree at most $n-1$.

From the Gaussian quadrature formula it follows that we may write

$$
P_{n}(z)=-\sum_{k=1}^{n} \lambda_{n, k} \frac{Q_{n}(z)}{t_{k}^{(n)}-z}=-Q_{n}(z) \int_{\alpha}^{\beta} \frac{d \phi_{n}(t)}{t-z},
$$

which means $P_{n}(z) / Q_{n}(z)=-\hat{\phi}_{n}(z)$. 
If the ESMP has a unique solution $\psi(t)$, then $\left\{\phi_{n}(t)\right\}$ converges to this solution, since otherwise convergent subsequences would produce distinct solutions. It follows that $\left\{\hat{\phi}_{n}(z)\right\}$ converges to $\hat{\psi}(z)$ for all $z \in \mathbf{C}-[\alpha, \beta]$. The sequence $\left\{P_{n}(z) / Q_{n}(z)\right\}$ is convergent for the same values of $z$.

We shall now show that if $\left\{P_{n}(z) / Q_{n}(z)\right\}$ converges on an interval of the form $\left(a_{\rho}, a_{\sigma}\right)$, where $\left(a_{\rho}, a_{\sigma}\right) \cap[\alpha, \beta]=\varnothing$, then the ESMP has a unique solution.

We note that the function

$$
\begin{aligned}
f(t) & =\frac{1}{(t-z)}\left[\frac{1}{N_{n-1}(t)}-\frac{1}{N_{n-1}(z)}\right] \\
& =\frac{N_{n-1}(z)-N_{n-1}(t)}{(t-z) N_{n-1}(t) N_{n-1}(z)}
\end{aligned}
$$

belongs to $R_{n-1}$, and thus $\Phi\left(Q_{n} \cdot f\right)=0$. This implies

$$
\begin{aligned}
\Phi_{t}( & \left.\frac{1}{t-z}\left[\frac{Q_{n}(t)}{N_{n-1}(t)}-\frac{Q_{n}(z)}{N_{n-1}(z)}\right]\right) \\
\quad= & \Phi_{t}\left(\frac{1}{t-z}\left[\frac{Q_{n}(t)-Q_{n}(z)}{N_{n-1}(z)}\right]\right)+\Phi_{t}\left(Q_{n}(t) f(t)\right) \\
& =\frac{1}{N_{n-1}(z)} P_{n}(z) .
\end{aligned}
$$

Hence

$$
P_{n}(z)=N_{n-1}(z) \Phi_{t}\left(\frac{1}{t-z}\left[\frac{Q_{n}(t)}{N_{n-1}(t)}-\frac{Q_{n}(z)}{N_{n-1}(z)}\right]\right) .
$$

We also note that the function

$$
g(t)=\frac{V_{n}(t)-V_{n}(z)}{(t-z) N_{n-1}(t)}
$$

belongs to $R_{n-1}$, and thus

$$
\Phi\left(Q_{n}(t) \frac{V_{n}(t)-V_{n}(z)}{(t-z) N_{n-1}(t)}\right)=0 .
$$

Let $\phi$ be an arbitrary solution of the EHMP. From (4.3) we obtain

$$
P_{n}(z)=N_{n-1}(z) \int_{-\infty}^{\infty} \frac{Q_{n}(t)}{(t-z) N_{n-1}(t)} d \phi(t)-Q_{n}(z) \hat{\phi}(z) .
$$

Similarly from (4.4) we get (noting that $N_{n}(t)=\left(t-a_{r}\right) N_{n-1}(t)$ )

$$
\int_{-\infty}^{\infty} \frac{Q_{n}(t)}{(t-z) N_{n-1}(t)} d \phi(t)=\frac{1}{V_{n}(z)} \int_{-\infty}^{\infty} \frac{Q_{n}(t)^{2}\left(t-a_{r}\right)}{t-z} d \phi(t) .
$$

Substitution of (4.6) into (4.5) and division by $Q_{n}(z)$ gives

$$
\frac{P_{n}(z)}{Q_{n}(z)}=\frac{1}{\left(z-a_{r}\right) Q_{n}(z)^{2}} \int_{-\infty}^{\infty} \frac{\left(t-a_{r}\right) Q_{n}(t)^{2}}{t-z} d \phi(t)-\hat{\phi}(z) .
$$

Now assume that $\phi$ is a solution of the ESMP, so that we may write

$$
-\hat{\phi}(z)=\frac{P_{n}(z)}{Q_{n}(z)}-\frac{1}{\left(z-a_{r}\right) Q_{n}(z)^{2}} \int_{\alpha}^{\beta} \frac{\left(t-a_{r}\right) Q_{n}(t)^{2}}{t-z} d \phi(t) .
$$


Let $z \in\left(a_{\rho}, a_{\sigma}\right)$ for some $\rho, \sigma$, where $\left(a_{\rho}, a_{\sigma}\right) \cap[\alpha, \beta]=\varnothing$. Then either $\left(t-a_{\rho}\right)$, $\left(t-a_{\sigma}\right)$, and $(t-z)$ are positive for all $t \in[\alpha, \beta]$, or $\left(t-a_{\rho}\right),\left(t-a_{\sigma}\right)$, and $(t-z)$ are negative for all $t \in[\alpha, \beta]$. The numbers $\left(z-a_{\rho}\right)$ and $\left(z-a_{\sigma}\right)$ have opposite sign. Therefore $\hat{\phi}(z)+P_{n}(z) / Q_{n}(z)$ and $\hat{\phi}(z)+P_{m}(z) / Q_{m}(z)$ have opposite sign when $n$ is of the form $n=p k+\rho$ and $m$ is of the form $m=p k+\sigma$. It follows that if the sequences $\left\{P_{p k+\rho}(z) / Q_{p k+\rho}(z): k=0,1,2, \ldots\right\}$ and $\left\{P_{p k+\sigma}(z) / Q_{p k+\sigma}(z)\right.$ : $k=0,1,2, \ldots\}$ are convergent and converge to the same value, then this value is $-\hat{\phi}(z)$. Thus if these two sequences converge to the same function on a subinterval of $\left(a_{\rho}, a_{\sigma}\right)$, then this function is $-\hat{\phi}(z)$. From the uniqueness of the Stieltjes transform of the solution follows uniqueness of the solution itself. (Recall that the transform is known on $\mathbf{C}-[\alpha, \beta]$ when it is known on an interval.)

We may sum up the results in this section as follows:

THEOREM 2. The following statements are equivalent.

(A) The ESMP has a unique solution.

(B) The sequence $P_{n}(z) / Q_{n}(z)$ converges on $\mathbf{C}-[\alpha, \beta]$.

(C) There exist an interval $\left(a_{\rho}, a_{\sigma}\right)$ disjoint from $[\alpha, \beta]$ such that the sequences $\left\{P_{p k+\rho}(z) / Q_{p k+\rho}(z): k=0,1,2, \ldots\right\}$ and $\left\{P_{p k+\sigma}(z) / Q_{p k+\sigma}(z): k=0,1,2, \ldots\right\}$ converge to the same value (depending on $z$ ) for every $z$ in some interval $(a, b) \subset$ $\left(a_{\rho}, a_{\sigma}\right)$.

\section{REFERENCES}

1. W. B. Jones and W. J. Thron, Survey of continued fraction methods of solving moment problems and related topics, Analytic Theory of Continued Fractions (Proceedings Loen, 1981), Lecture Notes in Math., vol. 932, Springer, Berlin, 1982, pp. 4-37.

2. W. B. Jones, W. J. Thron, and H. Waadeland, A strong Stieltjes moment problem, Trans. Amer. Math. Soc. 261 (1980), 503-528.

3. O. Njåstad, An extended Hamburger moment problem, Proc. Edinburgh Math. Soc. 28 (1985), 167-183.

4. $\_$Unique solvability of an extended Hamburger moment problem, J. Math. Anal. Appl. 124 (1987), 502-519.

5. __ Orthogonal rational functions with poles in a finite subset of R. II. Symposium on Orthogonal Polynomials and Their Applications, Segovia, 1986.

6. O. Perron, Die Lehre von den Kettenbrüchen, 3. Auflage, Band 2, Teubner, Stuttgart, 1957.

7. T. J. Stieltjes, Recherches sur les fractions continues, Ann. Fac. Sci. Toulouse Math. 8 (1894), 1-122.

Department of Mathematics, University of Trondheim-NTH, N-7034, TrondHEIM, NORWAY

Current address: Department of Mathematics, Campus Box 426, University of Colorado, Boulder, Colorado 80309 